\title{
https://doi.org/10.46813/2021-134-030 \\ HIGH-CURRENT RELATIVISTIC ELECTRON BEAM FOCUSING IN PLASMA
}

\section{O.V. Manuilenko, A.V. Pashchenko, V.G. Svichensky, B.V. Zajtsev \\ National Science Center “Kharkov Institute of Physics and Technology”, Kharkiv, Ukraine \\ E-mail:ovm@kipt.kharkov.ua}

The analysis of the envelope equation for high-current relativistic electron beam propagation in plasma in an external uniform magnetic field is presented. The envelope equation is obtained in a Hamiltonian form with an effective potential, which depends from electron beam and plasma parameters, and external magnetic field. Hamiltonian aproach allows fully analyze the behavior of the beam envelope as a function of the beam current, beam energy, plasma density and conductivity, as well as on the external magnetic field and the initial beam angular momentum.

PACS: 52.75.-d, 52.77.Fv, 52.80.Hc, 52.90.+z, 81.20.-n

\section{INTRODUCTION}

Different types of charged particle beam envelope equations in a plasma are widely used to analyze qualitative beam behavior under various operating conditions, in particular, during transportation of an ion or electron beam to a target, or an accelerating (slowing) structure [1 - 4]. The paper presents a analysis of the envelope equation for a high-current relativistic electron beam propagating in plasma in an external uniform magnetic field. In the paraxial approximation, for pure transport of a high-current electron beam in a plasma, the envelope equation can be presented in a Hamiltonian form with an effective potential $U_{\text {eff }}$, which depends from beam and plasma parameters, and external magnetic field. This allows fully analyze the behavior of the beam envelope as a function of the beam current, beam energy, plasma density and plasma conductivity, as well as on the external magnetic field and the initial beam angular momentum. There are four basic modes of beam evolution depending on the problem parameters: periodic beam radius oscillations, unlimited growth of the beam radius, growth of the beam radius in the process of its propagation in the plasma with initial radial velocity, and the pinch mode, when at some distance from the injection site the beam is compressed. Beam pinching length can be effectively controlled by plasma density and conductivity, as well as by external magnetic field. For beams with $I \sim 20 \mathrm{kA}, \mathrm{W} \sim 750 \mathrm{keV}$ [5], initial radius $r_{b o} \sim 1 \mathrm{~cm}$, charge neutralization factor $f_{e} \sim 1$, and current neutralization factor $f_{m}<<1$ pinching length $\sim 1 \mathrm{~cm}$. For pinching length $<<1 \mathrm{~cm}$, the paraxial approximation may not be applicable. In this case, it is necessary to solve numerically a complete system of equations that describes the beam envelope in a plasma, or use the particle-in-cell codes to simulate kinetic beam behaviour in a plasma. These simulations have shown that the pinch length can be effectively controlled by using the plasma density and its conductivity.

\section{BEAM ENVELOPE EQUATION. ANALYTICAL AND NUMERICAL ANALYSIS}

Consider an axially symmetric high-current relativistic electron beam propagating in plasma in an external uniform magnetic field $B_{z}$ directed along the beam axis. The motion equation for a charged particle with mass $m$ and charge $e$ in the transverse direction has the form:

$$
\dot{\vec{p}}_{\perp}=e\left(\vec{E}_{\perp}+(\vec{v} \times \vec{B})_{\perp} / c\right),
$$

where $\vec{p}_{\perp}=m \vec{v}_{\perp}, \gamma=\left(1-\beta^{2}\right)^{-1 / 2}, \beta=|\vec{v}| / c, \vec{v}$ is the particle velocity, $c$ is the speed of light, $\vec{v}_{\perp}=v_{r} \vec{e}_{r}+v_{\theta} \vec{e}_{\theta}, \vec{v}=\vec{v}_{\perp}+v_{z} \vec{e}_{z}, \quad \dot{p}_{\perp}=d \vec{p}_{\perp} / d t$. With the assumed cylindrical symmetry, the main field components are $E_{r}, B_{\theta}$, and external $B_{z}$. In this case the radial component of Eq. (1) yields

$$
\ddot{r}+\dot{r} \dot{\gamma} / \gamma-v_{\theta}^{2} / r=e\left(E_{r}+\left(v_{\theta} B_{z}-v_{z} B_{\theta}\right) / c\right) / m \gamma,
$$

and the azimuthal component of Eq. (1) yields a conservation of canonical angular momentum $P_{\theta}$ :

$$
\gamma r v_{\theta}+\Omega r^{2} / 2=P_{\theta} / m=\text { const }
$$

where $\Omega=e B_{z} / m c$. Eliminating $v_{\theta}$ from Eq. (2) using Eq. (3) gives the envelope equation:

$$
\ddot{r}_{b}+\frac{\dot{r}_{b} \dot{\gamma}}{\gamma}+\left(\frac{\Omega}{2 \gamma}\right)^{2} r_{b}=\frac{e}{\gamma m}\left[E_{r}-\beta_{z} B_{\theta}\right]+\left(\frac{P_{\theta}}{\gamma m}\right)^{2} \frac{1}{r_{b}^{3}},
$$

where $r_{b}$ is the transverse coordinate of a particle located at the outer beam boundary. The equation for $\gamma$ has the form: $\dot{\gamma}=e \vec{\beta} \cdot \vec{E} / m c$, where $\vec{\beta}=\vec{v} / c$, which in the paraxial approximation $\left(\left|v_{r}\right|,\left|v_{\theta}\right|<<v_{z}\right)$ can be presented as $\dot{\gamma}=e \beta_{z} E_{z} / m c$, where $\gamma \approx\left(1-\beta_{z}^{2}\right)^{-1 / 2}$.

The field components $E_{r}$ and $B_{\theta}$ at the edge of the beam are defined by Gauss' and Ampere's laws:

$$
\begin{aligned}
& \int_{S} \vec{E} \cdot \vec{N} d S=4 \pi e \int_{V} n_{b}\left(1-f_{e}\right) d V, \\
& \oint_{L} \vec{B} \cdot d \vec{L}=4 \pi e \int_{S} n_{b} \vec{\beta}\left(1-f_{m}\right) \cdot \vec{N} d S,
\end{aligned}
$$

where $n_{b}$ is the beam charge density, $\vec{j}_{b}=n_{b} \vec{v}$, and $f_{e}$, $f_{m}$ are the charge and current neutralization factors. For a beam of constant charge density out to the radius $r_{b}$ these give

$$
E_{r}=2 \pi e n_{b} r_{b}\left(1-f_{e}\right), \quad B_{\theta}=2 \pi e n_{b} r_{b} \beta_{z}\left(1-f_{m}\right) .
$$

Eq. (4), taking into account (5), can be written as [1]: 


$$
\ddot{r}_{b}+\frac{\dot{r}_{b} \dot{\gamma}}{\gamma}+\left(\frac{\Omega}{2 \gamma}\right)^{2} r_{b}-\frac{2 e I}{m \beta_{z} c \gamma^{3}}\left[1-\gamma^{2}\left(f_{e}-\beta_{z}^{2} f_{m}\right)\right] \frac{1}{r_{b}}=\left(\frac{P_{\theta}}{\gamma m}\right)^{2} \frac{1}{r_{b}^{3}},(6)
$$

where $I=\pi r_{b}^{2} n_{b} e \beta_{z} c$ is the beam current. Assuming that the charged particles beam moves with a constant longitudinal velocity ( $E_{z}=0$, pure transport of a beam in a plasma), Eq. (6) can be presented as:

$$
\ddot{r}_{b}+A r_{b}-\frac{B}{r_{b}}-\frac{C}{r_{b}^{3}}=0,
$$

where $A=(\Omega / 2 \gamma)^{2} ; B=2 e I\left[1-f_{e}-\beta_{z}^{2}\left(1-f_{m}\right)\right] / m \beta_{z} c \gamma$; $C=\left(P_{\theta} / \gamma m\right)^{2}$. In Eq. (7), the second term describes the effect of an external magnetic field on the beam envelope, the third term describes the effect of the intrinsic fields of a beam, partially compensated in charge and current, on its envelope, and, finally, the fourth term describes the influence of beam rotation on the beam envelope. Coefficient $A$ in (7) can be equal to zero if there is no external magnetic field, otherwise it is greater than zero, i.e., $A \in[0,+\infty[$. Coefficient $B$ in (7) can be greater than zero, equal to zero, or less than zero, depending on the combination of plasma $\left(f_{e}, f_{m}\right)$ and beam $\left(\beta_{z}\right)$ parameters, i.e., $\left.B \in\right]-\infty, 0,+\infty[$. Coefficient $C$ in (7) can be equal to zero, or greater than zero, i.e., $C \in[0,+\infty[$. Depending on the signs of the coefficients $A, B$ and $C$, or their equality (non-equality) to zero, the form of Eq. (7) changes. This means that the beam envelopes, which this equation describes, will also have different shapes. In general case, as shown below, the beam envelope can oscillate, constrict, or expand over time.

To analyze the possible behavior of the beam envelopes in plasma depending on the $A, B$, and $C$, it is convenient to represent Eq. (7) in Hamiltonian form. Eq. (7) can be obtained from the Hamiltonian $H\left(r_{b}, \dot{r}_{b}\right)=\dot{r}_{b}^{2} / 2+U\left(r_{b}\right)$, where the effective potential $U\left(r_{b}\right)$ has the form:

$$
U\left(r_{b}\right)=A r_{b}^{2} / 2-B \ln \left(r_{b}\right)+C / 2 r_{b}^{2} .
$$

In the expression for the effective potential (8), the first term describes the effect of an external magnetic field on the beam envelope, the second term describes the effect of the intrinsic fields of a beam partially compensated in charge and current on its envelope, and, the third term describes the effect of beam rotation on the envelope. As can be seen from (8), the shape of the effective potential $U\left(r_{b}\right)$ depends on the coefficients $A$, $B, C$. Depending on the form of the effective potential, the beam envelope behaves differently. If the effective potential $U\left(r_{b}\right)$ has a minimum at some value of $r_{b}$ and goes to positive infinity at $r_{b} \rightarrow 0$ and $r_{b} \rightarrow \infty$, as shown in Fig. 1,a, then the beam envelope should oscillate. If the effective potential $U\left(r_{b}\right)$ goes to positive infinity at $r_{b} \rightarrow 0$, and goes to zero or negative infinity at $r_{b} \rightarrow \infty$, as shown in Fig. 1,b, then the beam envelope should expand. If the effective potential $U\left(r_{b}\right)$ goes to negative infinity at $r_{b} \rightarrow 0$, and goes to positive infinity at $r_{b} \rightarrow \infty$, as shown in Fig. 1,c, then the beam envelope should compress.
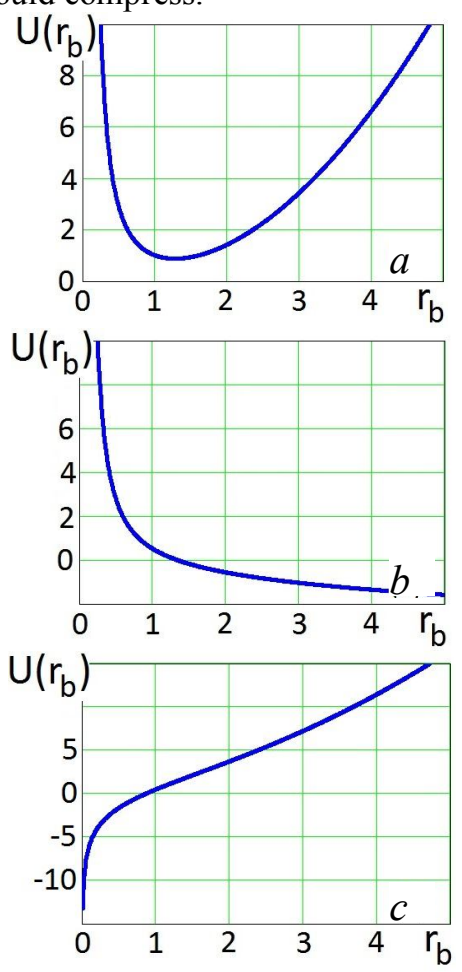

Fig. 1. The possible forms of the effective potential

$U\left(r_{b}\right)=A r_{b}^{2} / 2-B \ln \left(r_{b}\right)+C / 2 r_{b}^{2}$. Case (a) corresponds to the beam envelope oscillation, case (b) corresponds to the beam expansion, and case (c) corresponds to the beam compression

Fig. 2 shows the beam envelopes $r_{b}(t)$ for different shapes of the effective potential $U\left(r_{b}\right)$ which were obtained numerically by direct solution of Eq. (7). Fig. 2,a presents $r_{b}(t)$ for effective potential shown in Figs. 1,a; 2, b shows the beam envelope for $U\left(r_{b}\right)$ from Figs. 1,b; and 2,c presents $r_{b}(t)$ for $U\left(r_{b}\right)$ shown in Fig. 1,c.

In general case, since $A$ can either be equal to zero, or be greater than zero, $B$ can be greater than zero, equal to zero, or less than zero, and $C$ can either be equal to zero, or be greater than zero, there are 12 different combinations of $A, B, C$ coefficients, which determine the shape of effective potential $U\left(r_{b}\right)$. For cases $\{A>0, B>0, C>0\},\{A>0, B=0, C>0\}$, $\{A>0, B<0, C>0\}, \quad\{A=0, B<0, C>0\}$, $\{A>0, B>0, C=0\}$, the effective potential $U\left(r_{b}\right)$ has the shape as shown in Fig. 1,a, and the beam envelope oscillates. If $\{A=0, B>0, C>0\},\{A=0$, $B=0, C>0\},\{A=0, B>0, C=0\}$, the potential looks like in Fig. 1b, and the beam diverges. For $\{A>0, B<0, C=0\},\{A=0, B<0, C=0\}$, the potential $U\left(r_{b}\right)$ has the form as shown in Fig. 1,c, and the beam compresses. For $\{A=0, B=0, C=0\}$, the effective potential $U\left(r_{b}\right)=0$, and the Eq. (7) takes the form: $\ddot{r}_{b}=0 . A=0$ means that $B_{z}=0, \Omega=0$, and $P_{\theta}=m r r v_{\theta} . C=\left(P_{\theta} / \gamma_{o} m\right)^{2}=0$ means that $v_{\theta}=0$, and the beam expands, or contracts with an initial radial 
velocity $v_{r}$, which is usually much less than the initial velocity $v_{z}$. Thus, the beam propagates in plasma with an almost constant radius. A necessary condition for this is $B=0$, or $f_{e}=f_{e}^{e q}$, where $f_{e}^{e q}=\gamma^{-2}+\beta^{2} f_{m}$. In the case if $f_{m}<<1 /\left(\gamma^{2}-1\right)$, last term can be neglected, and $f_{e}^{e q}=\gamma^{-2}$, or $n_{p}=n_{b} \gamma^{-2}$, where $n_{p}$ is the plasma density. If $\{A>0, B=0, C=0\}$, the potential $U\left(r_{b}\right)=A r_{b}^{2} / 2$, and the Eq. (7) takes the form: $\ddot{r}_{b}+A r_{b}=0$. This case requires additional analysis at $r_{b} \rightarrow 0$.

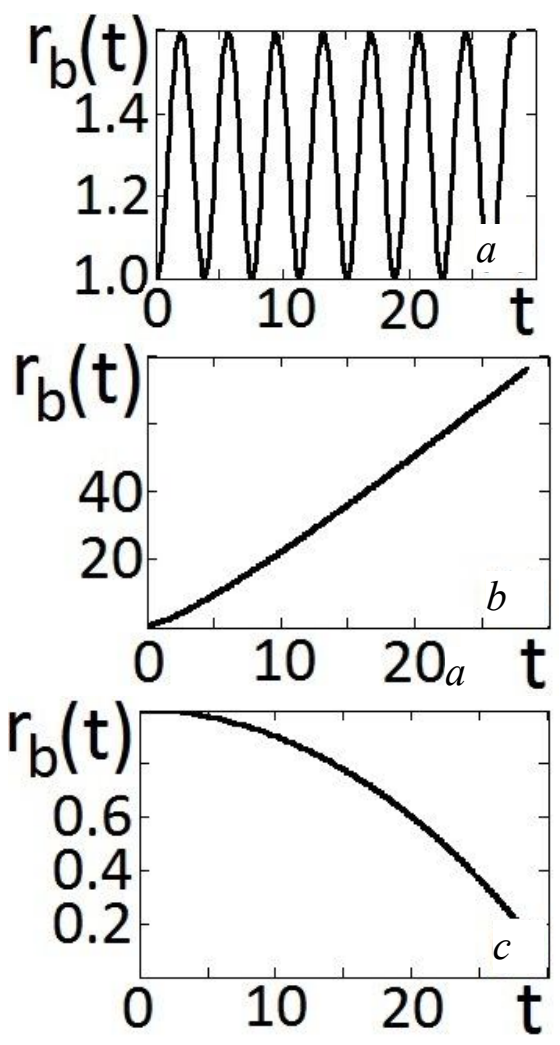

Fig. 2. The beam envelope as function of time for different shapes of the effective potential. Case (a) corresponds to the $U\left(r_{b}\right)$ shown in Fig. 1,a, case (b)

corresponds to the $U\left(r_{b}\right)$ shown in Fig. 1,b, and case (c) corresponds to the $U\left(r_{b}\right)$ shown in Fig. 1,c.

The envelopes $r_{b}(t)$ were obtained numerically by solving Eq. (7)

In the cases of the high-current relativistic electron beam pinching propagating in a plasma, the envelope equation (7) has the forms:

$$
r_{b}-\frac{2 e I}{m \beta_{z} c \gamma}\left[1-f_{e}-\beta_{z}^{2}\left(1-f_{m}\right)\right] \frac{1}{r_{b}}=0
$$

for $\{A=0, B<0, C=0\}$, and

$$
\ddot{r}_{b}+\left(\frac{\Omega}{2 \gamma}\right)^{2} r_{b}-\frac{2 e I}{m \beta_{z} c \gamma}\left[1-f_{e}-\beta_{z}^{2}\left(1-f_{m}\right)\right] \frac{1}{r_{b}}=0,
$$

for $\{A>0, B<0, C=0\}$. In both cases, in order for $B$ to be negative, it is necessary that $f_{e}>f_{e}^{e q}$.

Fig. 3 shows the numerical solution of Eq. (9) for the beam with the current $I=25 \mathrm{kA}$, initial radius $r_{b o}=$ $1 \mathrm{~cm}$, initial $v_{r o}=0, \gamma=2$, and $f_{e}=\{0.4,0.55,0.6\}$, which corresponds to plasma densities of the order of
$10^{12} \mathrm{~cm}^{-3}$, and $f_{m}=0$. As seen from Fig. 3, the focusing length $L_{f}$ is on the order of $2 \mathrm{~cm}$. It decreases with increasing charge neutralization factor $f_{e}$. This means that the $L_{f}$ can be controlled using $f_{e}$.

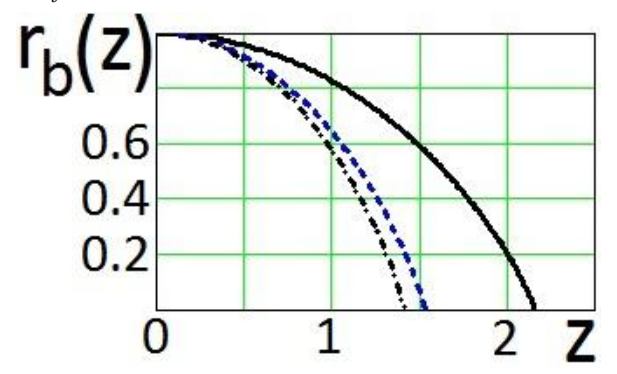

Fig. 3. Beam radius as function of longitudinal coordinate at various coefficients of charge neutralization of the beam in the plasma. $I=25 \mathrm{kA}, r_{b o}=1 \mathrm{~cm}$,

$v_{r o}=0, \gamma=2, f_{e}=\{0.4$ - black solid line,

0.55 - blue dotted line, 0.6 - black dash-dotted line\}.

$$
B_{z}=0, v_{\theta}=0 \text {. Lengths are in } \mathrm{cm}
$$

From (9), for the time $\tau$ during which the beam from the initial radius $r_{b o}$, at the initial $v_{r o}=0$, reaches the axis $\left(r_{b}(\tau)=0\right)$, the following expression can be obtained: $\tau=r_{b o} \sqrt{\pi / 2|B|}$. This allows to estimate the focusing length as $L_{f}=c \beta_{z} \tau$. For the parameters of beams and plasma from Fig. 3, focus lengths $L_{f}$ are $2.15 \mathrm{~cm}$ for $f_{e}=0.4,1.52 \mathrm{~cm}$ for $f_{e}=0.55$, and $1.41 \mathrm{~cm}$ for $f_{e}=0.6$. These coincide with the results of the numerical solution of Eq. (9) shown in Fig. 3.

Fig. 4 shows the numerical solution of Eq. (10) for a beam with $I=25 \mathrm{kA}, r_{b o}=1 \mathrm{~cm}, v_{r o}=0, \gamma=2$, and $f_{e}=\{0.4,0.55,0.6\}, f_{m}=0$. As seen from Fig. 4 , the focusing length $L_{f}$ is on the order of $1 \mathrm{~cm}$, it decreases with increasing charge neutralization factor $f_{e}$. The focusing length $L_{f}$ is also decreased due to the presence of an external magnetic field. This means that the $L_{f}$ can be controlled using $B_{z}$.

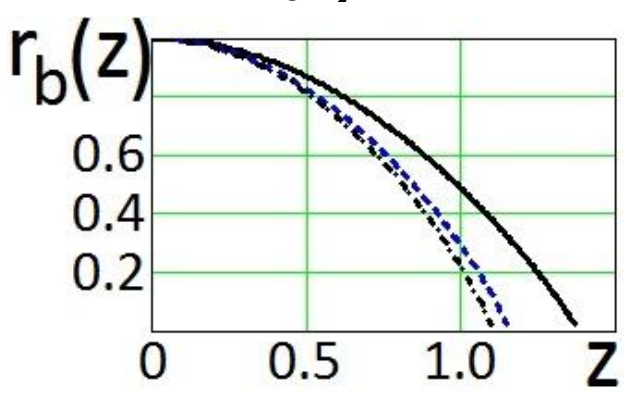

Fig. 4. Beam radius vs longitudinal coordinate at various coefficients of charge neutralization of the beam in the plasma. $I=25 \mathrm{kA}, r_{b o}=1 \mathrm{~cm}, v_{r o}=0, \gamma=2$, $f_{e}=\{0.4$ - black solid line, 0.55 - blue dotted line, 0.6 - black dash-dotted line $\} . B_{z}=5 k G s . v_{\theta} \neq 0$.

The rotation of the beam as a whole around the axis is necessary for $P_{\theta}=0$, and as a result, $C=0$. All lengths are in $\mathrm{cm}$ 
Fig. 5 shows the numerical solution of Eq. (7) where $\{A=0, B<0, C>0\}$ for a beam with $I=25 \mathrm{kA}$, $r_{b o}=1 \mathrm{~cm}, v_{r o}=0, \gamma=2$, and $f_{e}=\{0.4,0.55,0.6\}$, $f_{m}=0 . A=0$ and $C>0$ means that the beam as a whole rotates around the axis. It is seen that the beam is periodically focused by the plasma. The first focus is located at a distance of about $2 \mathrm{~cm}$ from the beam injection site, which is closed to the case shown in Fig. 3. This distance decreases with increasing charge neutralization factor $f_{e}$. The only difference between this case $\{A=0, B<0, C>0\}$, and the case shown in Fig. 3 $\{A=0, B<0, C=0\}$, is that $C>0$, i.e., the beam rotates as a whole around its axis. In the expression for the effective potential $U\left(r_{b}\right)$, this led to an additional term $C / 2 r_{b}^{2}$, which changed the shape of $U\left(r_{b}\right)$, turning the effective potential shown in Fig. 1,c to the potential shown in Fig. 1,a. This led to the oscillatory behavior of the beam envelope.

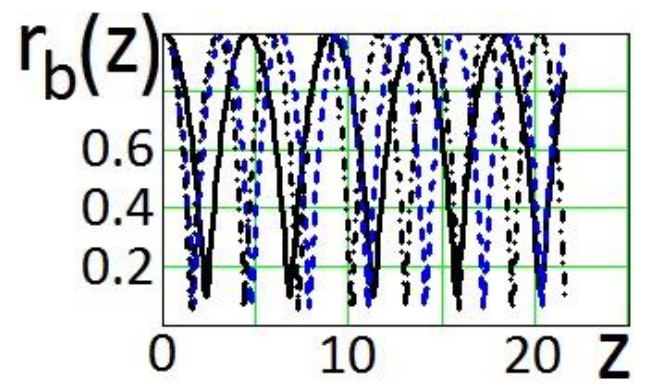

Fig. 5. Beam radius as function of longitudinal coordinate at various coefficients of charge neutralization of the beam in the plasma. $I=25 \mathrm{kA}, r_{b o}=1 \mathrm{~cm}$,

$v_{\text {ro }}=0, \gamma=2, f_{e}=\{0.4$ - black solid line; 0.55 - blue dotted line; 0.6 - black dash-dotted line\}.

$$
B_{z}=0, v_{\theta} \neq 0 \text {. Lengths are in } \mathrm{cm}
$$

To evaluate the applicability of the above model in experiments on focusing high-current electron beams in a plasma, a number of $2 \mathrm{~d} 3 \mathrm{v}$ particle-in-cell simulations were performed. The simulations involve solving a complete set of Maxwell's equations with chargeconserving schemes for calculating the current density on a mesh, and relativistic motion equations for charged particles in self-consistent electromagnetic fields. The XOOPIC code was used [6].

Fig. 6 shows, as example, simulation results for beam injection into a bounded plasma. The beam currents are $1 \mathrm{kA}$, and $10 \mathrm{kA}$. Other parameters are the same: $r_{b o}=1 \mathrm{~cm}, \gamma=2, f_{e}=1, v_{\theta}=0, B_{z}=0$. This is the case of Eq. (9) $-\{A=0, B<0, C=0\}$. The focal length estimates give: $L_{f}=4.8 \mathrm{~cm}$ for $I=1 \mathrm{kA}$, and $L_{f}=1.5 \mathrm{~cm}$ for $I=10 \mathrm{kA}$. Fig. 6,a,b clearly demonstrate these focuses with the little bit high focal lengths. Simulations showed that up to $I=50 \mathrm{kA}$, the ratio of the focal length obtained in particle-in-cell simulations to the $L_{f}$ is not high then 1.6. So, the envelope equations can be used for rough estimates of beam parameters in real experiments. Fig. 6,c,d shows electron beam density $n_{b}(r, z)$. It is seen that at the focus the beam densities sharply increase.
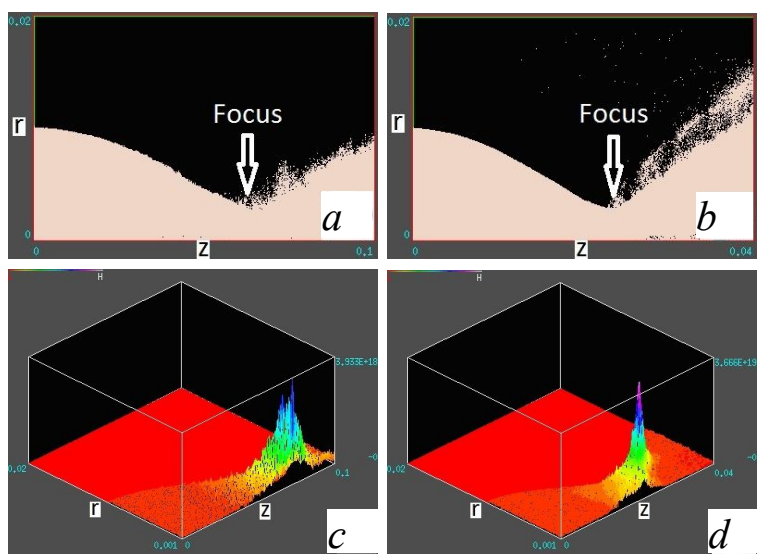

Fig. 6. Beam injection into a plasma: $a, b$-beam electrons distribution in $\{r, z\}$ plane; $c, d$-electron beam density $n_{b}(r, z) . I=1 \mathrm{kA}(a, c)$, and $I=10 \mathrm{kA}(b, d)$. $r_{b o}=1 \mathrm{~cm}, v_{r o}=0, \gamma=2, f_{e}=1, B_{z}=0, v_{\theta}=0$.

The dimensions of the simulation domain $z_{L} \times r_{L}$ were chosen based on solving the envelope equation (9) so that the focus is approximately in its center.

$L_{f}=4.8 \mathrm{~cm}$ for $I=1 \mathrm{kA}$, and $L_{f}=1.5 \mathrm{~cm}$ for

$I=10 \mathrm{kA} . z_{L} \times r_{L}=10 \times 2 \mathrm{~cm}$ for $I=1 \mathrm{kA}$, and $z_{L} \times r_{L}=4 \times 2 \mathrm{~cm}$ for $I=10 \mathrm{kA}$

\section{CONCLUSIONS}

Analysis of the envelope equation for a high-current relativistic electron beam propagating in a plasma in an external uniform magnetic field $B_{z}$ is presented. In the paraxial approximation the envelope equation has the Hamiltonian form $H\left(r_{b}, \dot{r}_{b}\right)=\dot{r}_{b}^{2} / 2+U\left(r_{b}\right)$ with the effective potential $U\left(r_{b}\right)=A r_{b}^{2} / 2-B \ln \left(r_{b}\right)+C / 2 r_{b}^{2}$, where $r_{b}$ is a beam radius, $A=(\Omega / 2 \gamma)^{2}$, $\Omega=e B_{z} / m c, \quad B=2 c^{2}\left(I / I_{A L}\right)\left[1-f_{e}-\beta^{2}\left(1-f_{m}\right)\right]$, $I_{A L}=\beta \gamma m c^{3} / e, I$ - beam current, $f_{e}, f_{m}$ - charge and current neutralization factors, $C=\left(P_{\theta} / \gamma m\right)^{2} / 2$, $P_{\theta}$ is the conserved angular momentum.

The analysis showed that, in the general case, four modes of beam propagation in plasma are possible: periodic beam radius oscillations, unlimited growth of the beam radius, growth of the beam radius with initial radial velocity, and the pinch mode, when at some distance from the injection site the beam is compressed. Beam pinching in a plasma occurs at length $L_{f}=c \beta r_{b o} \sqrt{\pi /|2 B|}, r_{b o}-$ initial beam radius. This length can be controlled by plasma density and conductivity, as well as by external magnetic field. Particle-incell simulations confirm these results.

\section{REFERENCES}

1. R.B. Miller. An introduction to the physics of intense charged particle beams. N-Y: "Plenum press", 1982.

2. E.P. Lee. Kinetic theory of a relativistic beam // Phys. Fluids. 1976, v. 19, № 1, p. 60-69. 
3. E.P. Lee, R.K. Cooper. General envelope equation for cylindrically symmetric charged-particle beams // Particle Accelerators. 1976, v. 7, p. 83-95.

4. V.I. Maslov, I.N. Onishchenko, I.P. Yarovaya. Fields excited and providing a uniform focusing of short relativistic electron bunches in plasma // East European Journal of Physics. 2014, v. 1, № 2, p. 9295.

5. A.B. Batrakov, E.G. Glushko, A.M. Yegorov, et al. Study of hard X-ray bremsstrahlung at the radiation- beam complex “TEMP” // Problems of Atomic Science and Technology. 2015, № 6, p. 100-104.

6. J.P. Verboncoeur, A.B. Langdon, N.T. Gladd. An object-oriented electromagnetic PIC code // Computer Physics Communications. 1995, v. 87, p. 199211.

Article received 17.06.2021

\section{ФОКУСИРОВКА СИЛЬНОТОЧНОГО РЕЛЯТИВИСТСКОГО ЭЛЕКТРОННОГО ПУЧКА В ПЛАЗМЕ}

\section{О.В. Мануйленко, А.В. Пащенко, В.Г. Свиченский, Б.В. Зайцев}

Представлен анализ уравнения огибающей для сильноточного релятивистского электронного пучка в плазме во внешнем однородном магнитном поле. Уравнение огибающей получено в гамильтоновой форме с эффективным потенциалом, который зависит от параметров электронного пучка и плазмы, а также от внешнего магнитного поля. Гамильтонов подход позволяет полностью проанализировать поведение огибающей пучка в зависимости от его тока и энергии, плотности и проводимости плазмы, а также от внешнего магнитного поля и начального углового момента пучка.

\section{ФОКУСУВАННЯ СИЛЬНОСТРУМОВОГО РЕЛЯТИВІСТСЬКОГО ЕЛЕКТРОННОГО ПУЧКА В ПЛАЗМІ}

\section{О.В. Мануйленко, А.В. Пащенко, В.Г. Свіченський, Б.В. Зайцев}

Представлено аналіз рівняння обвідної для потужнострумового релятивистского електронного пучка в плазмі в зовнішньому однорідному магнітному полі. Рівняння обвідної отримано в гамільтоновій формі 3 ефективним потенціалом, який залежить від параметрів електронного пучка і плазми, а також від зовнішнього магнітного поля. Гамільтонів підхід дозволяє повністю проаналізувати поведінку обвідного пучка в залежності від його струму, енергії, густини та провідності плазми, а також від зовнішнього магнітного поля $\mathrm{i}$ початкового кутового моменту пучка. 